\title{
THE PLETHYSTIC INVERSE OF THE ODD LIE REPRESENTATIONS
}

\author{
SHEILA SUNDARAM \\ In memory of my mother, Nirmala Sundaram
}

\begin{abstract}
The Frobenius characteristic of $\mathrm{Lie}_{n}$, the representation of the symmetric group $S_{n}$ afforded by the multilinear part of the free Lie algebra, is known to satisfy many interesting plethystic identities. In this paper we prove a conjecture of Richard Stanley establishing the plethystic inverse of the sum $\sum_{n \geq 0} L_{i e} e_{2 n+1}$ of the odd Lie characteristics. We obtain an apparently new plethystic decomposition of the regular representation of $S_{n}$ in terms of irreducibles indexed by hooks, and the Lie representations. We determine the plethystic inverse of the alternating sum of the odd Lie characteristics.

Keywords: Plethysm, plethystic inverse, free Lie algebra, Schur $P$-functions
\end{abstract}

\section{INTRODUCTION}

Let $\mathrm{Lie}_{n}$ denote the Frobenius characteristic of the representation of the symmetric group $S_{n}$ obtained by inducing the representation afforded by a primitive $n$th root of unity from the cyclic subgroup $C_{n}$ (generated by the long $n$-cycle) up to $S_{n}$. The symmetric functions $\mathrm{Lie}_{n}$ are known to arise in many different contexts, often involving plethystic identities. See, e.g. [8, Solutions to Ex. 7.88-7.89] and [12], [13]. In particular $L_{i} e_{n}$ describes the $S_{n}$-action on the multilinear component of the free Lie algebra on $n$ generators.

Let $e_{n}$ denote the elementary symmetric function of degree $n$; it is the Frobenius characteristic of the sign representation of $S_{n}$. In this paper we establish a plethystic identity conjectured by Richard Stanley, Theorem 1.1 below.

\section{Theorem 1.1.}

The symmetric functions $\frac{\sum_{n \geq 0} e_{2 n+1}}{\sum_{n \geq 0} e_{2 n}}$ and $\sum_{n \geq 0}$ Lie $e_{2 n+1}$ are plethystic inverses.

We also show that

\section{Theorem 1.2.}

The functions $\frac{\sum_{n \geq 0}(-1)^{n} e_{2 n+1}}{\sum_{n \geq 0}(-1)^{n} e_{2 n}}$ and $\sum_{n \geq 0}(-1)^{n}$ Lie $e_{2 n+1}$ are plethystic inverses.

The quotient of symmetric functions in Theorem 1.2 has been studied by other authors [2, 10]. The odd Lie representations also appear in [3] in connection with the free Jordan algebra.

Date: 2021 Dec 8.

2010 Mathematics Subject Classification. 05E10, 20 C30. 


\section{Preliminary identities}

We follow [5] and [8] for notation regarding symmetric functions.

In particular, $h_{n}, e_{n}$ and $p_{n}$ denote respectively the complete homogeneous, elementary and power-sum symmetric functions, and $\omega$ is the involution on the ring of symmetric functions which takes $h_{n}$ to $e_{n}$. If ch is the Frobenius characteristic map from the representation ring of the symmetric group $S_{n}$ to the ring of symmetric functions with real coefficients, then $h_{n}=\operatorname{ch}\left(1_{S_{n}}\right)$ is the characteristic of the trivial representation, and $e_{n}=\operatorname{ch}\left(\operatorname{sgn}_{S_{n}}\right)$ is the characteristic of the sign representation of $S_{n}$. Finally, for a partition $\mu$ of $n$, the irreducible $S_{n}$-module indexed by $\mu$ maps to the Schur function $s_{\mu}$ under the map ch.

If $q$ and $r$ are characteristics of representations of $S_{m}$ and $S_{n}$ respectively, they yield a representation of the wreath product $S_{m}\left[S_{n}\right]$ in a natural way, with the property that when this representation is induced up to $S_{m n}$, its Frobenius characteristic is the plethysm of $q$ with $r$, denoted $q[r]$. For more background about this operation, see [5]. We list the following key properties, in particular the fact that plethysm $(\cdot)[r]$ with a fixed symmetric function $r$ is an endomorphism (in the first argument) of the ring of symmetric functions [5, (8.3)]. See also [8, Chapter 7, Appendix 2, A2.6].

Proposition 2.1. If $q, r$ are symmetric functions of homogeneous degrees, $f, g, Y$ are arbitrary symmetric functions, and $\lambda$ is any partition, then

(1) $c[q]=c$ for any constant $c$.

(2) $p_{n}\left[p_{m}\right]=p_{n m}=p_{m}\left[p_{n}\right]$.

(3) $p_{n}[f]=f\left[p_{n}\right]$.

(4) $(f g)[q]=f[q] \cdot g[q]$; in particular $(c g)[q]=c \cdot(g[q])$ for any constant $c$.

(5) If $f g=X$, then $\left(\frac{f}{X}\right)[q]=g[q]=\frac{f[q]}{X[q]}$. In particular $\left(\frac{1}{f}\right)[q]=\frac{1}{f[q]}$.

(6) $f([g[Y]])=(f[g])[Y]$, i.e. plethysm is associative.

(7) $s_{\lambda}[q+r]=\sum_{\mu \subseteq \lambda} s_{\lambda / \mu}[q] s_{\mu}[r]$.

Here we single out the special cases $\lambda=(n), \lambda=\left(1^{n}\right)$ :

$h_{n}[q+r]=\sum_{k=0}^{n} h_{k}[q] h_{n-k}[r]$ and $e_{n}[q+r]=\sum_{k=0}^{n} e_{k}[q] e_{n-k}[r]$.

(8) $q[-r]=(-1)^{\operatorname{deg} q}(\omega q)[r]$.

(9) $\omega(q[r])=\left(\omega^{\operatorname{deg} r}(q)\right)[\omega r]$.

(10) $f[g]=p_{1} \Longleftrightarrow g[f]=p_{1}$.

Define

$$
\begin{aligned}
& H(t)=\sum_{i \geq 0} t^{i} h_{i}, \quad E(t)=\sum_{i \geq 0} t^{i} e_{i} ; \\
& H=\sum_{i \geq 0} h_{i}, \quad E=\sum_{i \geq 0} e_{i} .
\end{aligned}
$$

Recall the following well-known facts about the series $H(t)$ and $E(t)$. Parts $(3)$ and (4) are immediate consequences of (1) and (2), and Part (5) follows from the Pieri rule.

Proposition 2.2. ([5], 8])

(1) $H(t)=\exp \left(\sum_{k \geq 1} t^{k} p_{k} / k\right)$;

(2) $E(t)=\exp \left(\sum_{k \geq 1}(-1)^{k-1} t^{k} p_{k} / k\right)$;

(3) $H(t) E(-t)=1$; 
(4) $H(t) E(t)=\exp \left(\sum_{k \geq 1, k \text { odd }} 2 t^{k} p_{k} / k\right)$;

(5) $H(t) E(t)=1+2 \sum_{n \geq 1} t^{n} H k_{n}$, where we have written $H k_{n}$ for the sum of Schur functions corresponding to hook shapes $\sum_{k=0}^{n-1} s_{\left(n-k, 1^{k}\right)}$.

We refer the interested reader to [6] for background about the free Lie algebra, although our use of the Lie representation requires no knowledge other than what is presented here.

Define $\mathrm{Lie}_{n}$ to be the Frobenius characteristic of the representation of $S_{n}$ afforded by the multilinear component of the free Lie algebra on $n$ generators. It is well known that

$$
\operatorname{Lie}_{n}=\frac{1}{n} \sum_{d \mid n} \mu(d) p_{d}^{\frac{n}{d}}
$$

where $\mu$ is the number-theoretic Möbius function. This reflects the fact that $\operatorname{Lie}_{n}$ is the Frobenius characteristic of the $S_{n}$-module obtained by inducing a faithful irreducible representation of the cyclic subgroup $C_{n}$ (generated by the long $n$-cycle) up to $S_{n}$.

In [12, Theorem 3.2], [13, Theorem 5.8] it was shown that the following plethystic identities satisfied by $\mathrm{Lie}_{n}$ are equivalent, and a uniform derivation of the identities was provided. The first identity is a theorem of Thrall [15]. The equivalence of the second identity with the first is a consequence of [13, Proposition 6.6]. See also [14].

Theorem 2.3. The following identities hold:
(1) $H[\operatorname{Lie}(t)]=\frac{1}{1-t p_{1}}$.
(2) $E[\operatorname{Lie}(t)]=\frac{1-t^{2} p_{2}}{1-t p_{1}}$.

Definition 2.4. If $F=\sum_{n \geq 0} f_{n}$ is any formal power series of symmetric functions $\left\{f_{n}\right\}$ where $f_{n}$ is homogeneous of degree $n$, we will write

$$
F_{\text {odd }}=f_{1}+f_{3}+\cdots=\sum_{n \geq 0} f_{2 n+1}, \quad F_{\text {even }}=f_{0}+f_{2}+f_{4}+\cdots=\sum_{n \geq 0} f_{2 n} .
$$

We also write, for an indeterminate $t, F(t)=\sum_{n \geq 0} t^{n} f_{n}$, and

$F_{\text {odd }}(t)=t f_{1}+t^{3} f_{3}+\cdots=\sum_{n \geq 0} t^{2 n+1} f_{2 n+1}, \quad F_{\text {even }}(t)=f_{0}+t^{2} f_{2}+t^{4} f_{4}+\cdots=\sum_{n \geq 0} t^{2 n} f_{2 n}$.

The following observations will be useful for our arguments.

Proposition 2.5. We have

(1) $H_{\text {odd }}(t) E_{\text {even }}(t)-H_{\text {even }}(t) E_{\text {odd }}(t)=0$.

(2) $H_{\text {even }}(t) E_{\text {even }}(t)-H_{\text {odd }}(t) E_{\text {odd }}(t)=1$.

(3) $H_{\text {odd }}(t)=\frac{H(t) E(t)-1}{2 E(t)}, H_{\text {even }}(t)=\frac{H(t) E(t)+1}{2 E(t)}$.

Proof. Parts (1) and (2) follow from Proposition 2.2 by isolating the terms of even and odd degree of the power series in $H(t) E(-t)=1$. Part (3) follows from the fact that

$$
2 H_{\text {odd }}(t)=H(t)-H(-t)=H(t)-\frac{1}{E(t)},
$$

with the corresponding statement for $H_{\text {even }}(t)$. 
Proposition 2.6. The symmetric function $\frac{H_{\text {odd }}(t)}{H_{\text {even }}(t)}=\frac{E_{\text {odd }}(t)}{E_{\text {even }}(t)}$ is invariant under the involution $\omega$, and is equal to each of the following:

(1) $\frac{H(t) E(t)-1}{H(t) E(t)+1}$

(2) $\frac{\sum_{n \geq 1} t^{n} H k_{n}}{1+\sum_{n \geq 1} t^{n} H k_{n}}$, where $H k_{n}$ is the sum of the $n$ Schur functions corresponding to hook shapes of size $n$

(3) $\tanh \left(\sum_{k \geq 0} \frac{t^{2 k+1} p_{2 k+1}}{2 k+1}\right)$. This in turn equals $\sum_{n \geq 0}(-1)^{n} E_{2 n+1} \frac{Z^{2 n+1}}{(2 n+1) !}$, where we have written $Z=\sum_{k \geq 0} \frac{t^{2 k+1} p_{2 k+1}}{2 k+1}$, and $E_{2 n+1}$ is the tangent number.

Proof. Parts (1) and (2) are clear from Part (3) of Proposition 2.5] and Part (5) of Proposition 2.2.

For Part (3), we use Part (4) of Proposition 2.2. Writing $\theta=\sum_{k \text { odd }} \frac{t^{k} p_{k}}{k}$, the function in Part (1) equals $\frac{e^{2 \theta}-1}{e^{2 \theta}+1}=\tanh \theta$, as claimed. The invariance under $\omega$ is clear. The last statement is a consequence of the generating function [7] for the tangent numbers $E_{2 n+1}$.

\section{Proofs of Theorem 1.1 and Theorem 1.2}

The crux of the proof of Theorem 1.1 lies in the following:

Proposition 3.1. We have the plethystic identity

$$
\sum_{k \text { odd }} \frac{p_{k}}{k}\left[\text { Lie }_{\text {odd }}\right]=\sum_{m \text { odd }} \frac{p_{1}^{m}}{m} .
$$

Proof. Let $\delta_{1, j}$ denote the Kronecker $\delta$, which is 1 if $j=1$ and zero otherwise. Using the properties listed in Proposition 2.1, in particular Parts (2) and (3), we obtain

$$
\begin{aligned}
& \sum_{k \geq 1, k \text { odd }} \frac{p_{k}}{k}\left[\sum_{n \text { odd }} \frac{1}{n} \sum_{d \mid n} \mu(d) p_{d}^{\frac{n}{d}}\right]=\sum_{k \geq 1, k \text { odd }} \frac{1}{k} \sum_{n \text { odd }} \frac{1}{n} \sum_{d \mid n} \mu(d) p_{d}^{\frac{n}{d}}\left[p_{k}\right] \\
= & \sum_{\substack{k, n \geq 1 \\
k, n \text { odd }}} \frac{1}{k n} \sum_{d \mid n} \mu(d) p_{k d}^{\frac{n}{d}}=2 \sum_{\substack{k, m, d \geq 1 \\
k, m, d \text { odd }}} \frac{1}{k m d} \mu(d) p_{k d}^{m}
\end{aligned}
$$

where we have put $n=m d$;

$$
\begin{aligned}
& =\sum_{\substack{m, j \geq 1 \\
m, j \text { odd }}} \frac{1}{j m} \sum_{d \mid j} \mu(d) p_{j}^{m} \text { where we have put } j=k d ; \\
& =\sum_{\substack{m, j \geq 1 \\
m, j \text { odd }}} \frac{p_{j}^{m}}{j m} \sum_{d \mid j} \mu(d)=\sum_{\substack{m \geq 1 \\
m \text { odd }}} \frac{p_{1}^{m}}{m}, \text { since } \sum_{d \mid j} \mu(d)=\delta_{1, j} \\
& =\frac{1}{2} \log \frac{1+p_{1}}{1-p_{1}} .
\end{aligned}
$$


Proof of Theorem 1.1. Take the plethysm of Part (3) of Proposition 2.6 (for $t=1$ ) with Lie $_{\text {odd }}$. Associativity of the plethysm operation gives

$$
\tanh \left(\sum_{k \geq 0} \frac{p_{2 k+1}}{2 k+1}\right)\left[\text { Lie }_{\text {odd }}\right]=\tanh \left(\sum_{m \text { odd }} \frac{p_{1}^{m}}{m}\right) .
$$

The right-hand side of the identity in Proposition 3.1 is simply $\operatorname{arctanh} p_{1}$ as a formal power series. Hence the above calculation reduces to $\tanh \left(\operatorname{arctanh}\left(p_{1}\right)\right)=p_{1}$, as claimed.

We now present a restatement of Theorem 1.1 that is of independent interest.

Lemma 3.2. The plethystic identity of Theorem 1.1] is equiivalent to the following identity:

$$
(H E)\left[\text { Lie }_{\text {odd }}\right]=\frac{1+p_{1}}{1-p_{1}}
$$

Proof. Using the fact that plethysm with $g$ is a ring homomorphism, specifically Proposition 2.1, Parts (4) and (5), in conjunction with Part (1) of Proposition 2.6, we may restate Theorem 1.1 as

$$
\frac{E_{\text {odd }}\left[\text { Lie }_{\text {odd }}\right]}{E_{\text {even }}\left[\text { Lie }_{\text {odd }}\right]}=\frac{(H E)\left[\text { Lie }_{\text {odd }}\right]-1}{(H E)\left[\text { Lie }_{\text {odd }}\right]+1}=p_{1} .
$$

Rearranging, we see that Theorem 1.1 is equivalent to

$$
(H E)\left[\text { Lie }_{\text {odd }}\right]-1=p_{1} \cdot\left((H E)\left[\text { Lie }_{\text {odd }}\right]+1\right) \text {, giving Eqn. (3.2). }
$$

Identity (3.2) leads to the following reformulation of Theorem 1.1; compare with Thrall's decomposition of the regular representation in Theorem 2.3 .

Proposition 3.3. The regular representation admits the plethystic decomposition

$$
p_{1}^{n}=\left.H k\left[\text { Lie }_{\text {odd }}\right]\right|_{\operatorname{deg} n},
$$

where $H k$ is the sum of all hooks, $H k=s_{(1)}+\sum_{n \geq 2} \sum_{r=0}^{n-1} s_{\left(n-r, 1^{r}\right)}$.

Proof. The sum of hooks enters the left-hand side of Eqn. (3.2) via Part (5) of Proposition [2.2, giving

$$
1+2 H k\left[\text { Lie }_{\text {odd }}\right]=1+2 \sum_{n \geq 1} p_{1}^{n}
$$

which is as claimed.

It would be interesting to find a representation-theoretic interpretation of the above decomposition.

The following lemma is easily deduced from Proposition 2.1, Part (7), or from Proposition 2.2, Part (1); see also [12].

Lemma 3.4. For any series of symmetric functions $F$ and $G, H[F+G]=H[F] \cdot H[G]$, $E[F+G]=E[F] \cdot E[G]$, and thus $(H E)[F+G]=(H E)[F] \cdot(H E)[G]$.

From identity (3.2) we also see that

\section{Proposition 3.5.}

$$
(H E)\left[\text { Lie }_{\text {even }}\right]=\left(1-p_{2}\right)\left(1-p_{1}\right)^{-2}=\left(1-p_{1}\right)^{-1} E[\text { Lie }]
$$


Proof. Writing Lie $=L_{i e_{\text {odd }}}+L_{i e_{\text {even }}}$ and using Lemma 3.4 and Theorem 2.3, we have the identities

$$
\begin{gathered}
H\left[\text { Lie }_{\text {odd }}\right] \cdot H\left[\text { Lie }_{\text {even }}\right]=\left(1-p_{1}\right)^{-1}, \\
E\left[\text { Lie }_{\text {odd }}\right] \cdot E\left[\text { Lie }_{\text {even }}\right]=\left(1-p_{2}\right)\left(1-p_{1}\right)^{-1}
\end{gathered}
$$

Write $A=(H E)\left[\right.$ Lie $\left._{\text {odd }}\right], B=(H E)\left[\right.$ Lie $\left._{\text {even }}\right]$.

Using the fact that plethysm is a ring homomorphism in the first argument, we have

$$
A \cdot B=(H E)\left[\text { Lie }_{\text {odd }}\right] \cdot(H E)\left[\text { Lie }_{\text {even }}\right]=\left(1-p_{2}\right)\left(1-p_{1}\right)^{-2} .
$$

Since $A=\frac{1+p_{1}}{1-p_{1}}$ from Eqn. (3.2) , the result follows. The second equality is a consequence of Part (2) of Theorem 2.3 .

Next we address Theorem 1.2. For this we need to establish analogues of Propositions 2.6 and 3.1. In order to prove the identities below, we work with the ring of symmetric functions with coefficients in $\mathbb{C}[t]$.

We have the following analogues of Propositions 2.5] and 2.6. Recall that we write $H_{\text {odd }}^{\text {alt }}$ for the alternating sum $\sum_{k \geq 0}(-1)^{k} h_{2 k+1}$, and $H_{\text {even }}^{\text {alt }}$ for the alternating sum $\sum_{k \geq 0}(-1)^{k} e_{2 k}$, and similarly for $E$.

Proposition 3.6. We have

(1) $H(i t)=H_{\text {even }}^{\text {alt }}(t)+i H_{\text {odd }}^{\text {alt }}(t)$;

(2) $E(i t)=E_{\text {even }}^{\text {alt }}(t)+i E_{\text {odd }}^{\text {alt }}(t)$;

(3) $H_{\text {odd }}^{\text {alt }}(t) E_{\text {even }}^{\text {alt }}(t)-H_{\text {even }}^{\text {alt }}(t) E_{\text {odd }}^{\text {alt }}(t)=0$. In particular we have $\left(1-h_{2}+h_{4}-\ldots\right)\left(e_{1}-e_{3}+e_{5}-\ldots\right)=\left(h_{1}-h_{3}+h_{5}-\ldots\right)\left(1-e_{2}+e_{4}-\ldots\right)$.

(4) $H_{\text {even }}^{\text {alt }}(t) E_{\text {even }}^{\text {alt }}(t)+H_{\text {odd }}^{\text {alt }}(t) E_{\text {odd }}^{\text {alt }}(t)=1$. In particular, we have

$$
\left(1-h_{2}+h_{4}-\ldots\right)\left(1-e_{2}+e_{4}-\ldots\right)=1-\left(h_{1}-h_{3}+h_{5}-\ldots\right)\left(e_{1}-e_{3}+e_{5}-\ldots\right) .
$$

Proof. This follows easily as in the proof of Proposition 2.5 , since $H(i t) E(-i t)=1$.

We will need the following expressions involving the alternating sum of the hooks $H k_{n}$ defined previously. Let

$$
H k_{\text {even }}^{\text {alt }}(t)=\sum_{n \geq 0, n \text { even }}(-1)^{\frac{n}{2}} t^{n} H k_{n}, \quad H k_{\text {odd }}^{\text {alt }}(t)=\sum_{n \geq 1, n \text { odd }}(-1)^{\frac{n-1}{2}} t^{n} H k_{n} .
$$

Proposition 3.7. The symmetric function $\frac{H_{\text {odd }}^{\text {alt }}(t)}{H_{\text {even }}^{\text {alt }}(t)}=\frac{E_{\text {odd }}^{\text {alt }}(t)}{E_{\text {even }}^{\text {alt }}(t)}$ is invariant under the involution $\omega$, and equals each of the following.

(1) $\frac{H(i t) E(i t)-1}{i(H(i t) E(i t)+1)}$;

(2) $\frac{H k_{\text {odd }}^{\text {alt }}(t)}{\left(H k_{\text {even }}^{\text {alt }}(t)\right)^{2}+\left(H k_{\text {odd }}^{\text {alt }}(t)\right)^{2}}$;

furthermore $\left(H k_{\text {odd }}^{\text {alt }}(t)\right)^{2}=H k_{\text {even }}^{\text {alt }}(t)-\left(H k_{\text {even }}^{\text {alt }}(t)\right)^{2}$.

(3) $\tan \left(\sum_{k \geq 0} \frac{(-1)^{k} t^{2 k+1} p_{2 k+1}}{2 k+1}\right)$. This in turn equals $\sum_{n \geq 0} E_{2 n+1} \frac{W^{2 n+1}}{(2 n+1) !}$, where we have written $W=\sum_{k \geq 0} \frac{(-1)^{k} t^{2 k+1} p_{2 k+1}}{2 k+1}$, and $E_{2 n+1}$ is the tangent number. 
Proof. The equality of the two quotients is immediate from Part (3) of the preceding proposition, and the invariance under $\omega$ then follows.

We have $2 i H_{\text {odd }}^{\text {alt }}(t)=H(i t)-H(-i t), \quad 2 H_{\text {even }}^{\text {alt }}(t)=H(i t)+H(-i t)$. Since $H(-i t) E(i t)=$ 1 , we obtain the expression in Part (1).

For simplicity put $X=H k_{\text {even }}^{\text {alt }}(t), Y=H k_{\text {odd }}^{\text {alt }}(t)$. From Part (5) of Proposition 2.2, we see that

$$
(H E)(i)-1=2(X+i Y-1),(H E)(i)+1=2(X+i Y),
$$

and hence the expression in Part (1) is converted to

$$
-i\left(1-(X+i Y)^{-1}\right)=\frac{Y}{X^{2}+Y^{2}}-i \frac{X^{2}+Y^{2}-X}{X^{2}+Y^{2}} .
$$

The imaginary part is zero (since $\frac{E_{\text {odd }}^{\text {alt }}(t)}{E_{\text {even }}^{\text {alt }}(t)}$ has real coefficients), yielding Part $(2)$.

Using Part (4) of Proposition 2.2, we have, writing $\theta=\sum_{k \geq 1, k \text { odd }} \frac{(-1)^{\frac{k-1}{2}} t^{k} p_{k}}{k}$,

$$
(H E)(i t)=H(i t) E(i t)=\exp \sum_{\substack{k \geq 1 \\ k \text { odd }}} \frac{2 i^{k} t^{k} p_{k}}{k}=e^{2 i \theta},
$$

Part (3) now follows, since the quotient in Part (1) equals $\frac{e^{2 i \theta}-1}{i\left(e^{2 i \theta}+1\right)}=\tan (\theta)$. The last statement is a consequence of the generating function for the tangent numbers $E_{2 n+1}$, [7].

We are now ready to prove Theorem 1.2. The analogue of Proposition 3.1 is:

Proposition 3.8. The following plethystic identity holds:

$$
\sum_{k \text { odd }}(-1)^{\frac{k-1}{2}} \frac{p_{k}}{k}\left[\text { Lie }_{\text {odd }}^{\text {alt }}\right]=\sum_{m \text { odd }}(-1)^{\frac{m-1}{2}} \frac{p_{1}^{m}}{m} .
$$

Proof. The left-hand side of (3.6) is

$$
\begin{aligned}
& \sum_{k \geq 1, k \text { odd }}(-1)^{\frac{k-1}{2}} \frac{p_{k}}{k}\left[\sum_{n \text { odd }}(-1)^{\frac{n-1}{2}} \frac{1}{n} \sum_{d \mid n} \mu(d) p_{d}^{\frac{n}{d}}\right] \\
= & \sum_{k \geq 1, k \text { odd }}(-1)^{\frac{k-1}{2}} \frac{1}{k} \sum_{n \text { odd }}(-1)^{\frac{n-1}{2}} \frac{1}{n} \sum_{d \mid n} \mu(d) p_{d}^{\frac{n}{d}}\left[p_{k}\right] \\
& \sum_{k, n \text { odd }} \frac{1}{k n}(-1)^{\frac{k-1}{2}}(-1)^{\frac{n-1}{2}} \sum_{d \mid n} \mu(d) p_{k d}^{\frac{n}{d}}=\sum_{\substack{k, m, d \geq 1 \\
k, m, d \text { odd }}} \frac{1}{k m d}(-1)^{\frac{k-1}{2}}(-1)^{\frac{m d-1}{2}} \mu(d) p_{k d}^{m}
\end{aligned}
$$

where we have put $n=m d$;

$$
\begin{aligned}
& =\sum_{\substack{m, j \geq 1 \\
m, j \text { odd }}} \frac{1}{j m} \sum_{d \mid j}(-1)^{\frac{j / d-1}{2}}(-1)^{\frac{m d-1}{2}} \mu(d) p_{j}^{m} \text { where we have put } j=k d ; \\
& =\sum_{\substack{m, j \geq 1 \\
m, j \text { odd }}} \frac{p_{j}^{m}}{j m} \sum_{d \mid j}(-1)^{\frac{j / d+m d}{2}}(-1) \mu(d) ; \text { note that } j / d+m d \text { is even. }
\end{aligned}
$$


Since $d$ is always odd, we have $(-1)=(-1)^{d}$ and the inner sum can be rewritten as

$$
\begin{aligned}
& \sum_{d \mid j}(-1)^{\frac{j+m d^{2}}{2}}(-1) \mu(d)=\sum_{d \mid j}(-1)^{\frac{(j-1)+\left(m d^{2}-1\right)}{2}} \mu(d) \\
& =(-1)^{\frac{j-1}{2}}(-1)^{\frac{m-1}{2}} \sum_{d \mid j} \mu(d)(-1)^{\frac{m\left(d^{2}-1\right)}{2}}=(-1)^{\frac{j-1}{2}}(-1)^{\frac{m-1}{2}} \sum_{d \mid j} \mu(d),
\end{aligned}
$$

since, for $d$ odd, $d^{2}-1=(d-1)(d+1) \equiv 0 \bmod 4$.

But $\sum_{d \mid j} \mu(d)$ is 1 if $j=1$ and 0 otherwise, making the left hand side of (3.6) equal to

$$
\sum_{\substack{m \geq 1 \\ m \text { odd }}}(-1)^{\frac{m-1}{2}} \frac{p_{1}^{m}}{m}
$$

as claimed.

Proof of Theorem 1.2. Part (3) of Proposition 3.7 gives, as before by associativity,

$$
\frac{E_{\text {odd }}^{\text {alt }}}{E_{\text {even }}^{\text {alt }}}\left[\text { Lie } e_{\text {odd }}^{\text {alt }}\right]=\tan \left(\sum_{k \text { odd }}(-1)^{\frac{k-1}{2}} \frac{p_{k}}{k}\left[\text { Lie }_{\text {odd }}^{\text {alt }}\right]\right)=\tan \left(\sum_{\substack{m \geq 1 \\ m \text { odd }}}(-1)^{\frac{m-1}{2}} \frac{p_{1}^{m}}{m}\right) .
$$

Clearly the argument of the tangent function is $\arctan p_{1}$, so the plethysm equals $p_{1}$.

Exactly as in the previous case, Theorem 1.2 has an equivalent formulation which also gives an analogue of Proposition 3.3.

Proposition 3.9. We have the identities

$$
\left.\sum_{m \geq 2, m \text { even }}(-1)^{\frac{m}{2}-1} H k_{m}\left[\text { Lie } e_{\text {odd }}^{\text {alt }}\right]\right|_{\operatorname{deg} 2 n}=(-1)^{n} p_{1}^{2 n}, n \geq 1,
$$

and

$$
\left.\sum_{m \geq 1, m \text { odd }}(-1)^{\frac{m-1}{2}} H k_{m}\left[\text { Lie } e_{\text {odd }}^{\text {alt }}\right]\right|_{\operatorname{deg} 2 n+1}=(-1)^{n} p_{1}^{2 n+1}, n \geq 0 .
$$

Proof. Using Part (1) of Proposition 3.7 and rearranging the plethysm, Theorem 1.2 has the equivalent formulation

$$
(H E)(i)\left[\operatorname{Lie}_{\text {odd }}^{\text {alt }}\right]-1=i p_{1} \cdot\left((H E(i))\left[\text { Lie }_{\text {odd }}^{\text {alt }}\right]+1\right),
$$

or

$$
(H E)(i)\left[\text { Lie } e_{\text {odd }}^{a l t}\right]=\frac{1+i p_{1}}{1-i p_{1}} .
$$

In the proof of Proposition 3.7, put $t=1$ in Eqn. (3.5). We obtain

$$
H E(i)=\left(2 X^{\prime}-1\right)+2 i Y^{\prime}
$$

for $X^{\prime}=H k_{\text {even }}^{\text {alt }}(1)=\sum_{m \geq 0, m \text { even }}(-1)^{\frac{m}{2}} H k_{m}, Y^{\prime}=H k_{\text {odd }}^{\text {alt }}(1)=\sum_{m \geq 1, m \text { odd }}(-1)^{\frac{m-1}{2}} H k_{m}$, and hence we have the identities

$$
\left(2 X^{\prime}-1\right)\left[\text { Lie } e_{\text {odd }}^{\text {alt }}\right]=1-\frac{2 p_{1}^{2}}{1+p_{1}^{2}}, \quad\left(2 Y^{\prime}\right)\left[\text { Lie } e_{\text {odd }}^{\text {alt }}\right]=\frac{2 p_{1}}{1+p_{1}^{2}} .
$$

Noting that $2 X^{\prime}-1=1+2 \sum_{m \geq 2, m \text { even }}(-1)^{\frac{m}{2}} H k_{m}$, the result follows. 
As mentioned in the Introduction, the quotient of symmetric functions $\frac{E_{\text {odd }}^{\text {alt }}}{E_{\text {even }}^{\text {alt }} \text { has }}$ already appeared in the literature. The earliest reference known to us, albeit implicitly, is a 1973 paper of Carlitz [2]. See also [8, Solution to Exercise 7.64 (c)], [10, Theorem 2.2 and the note on Page 4] and [1, Proposition 3.4]. Carlitz gives the following skew-Schur function expansion for the quotient $\frac{E_{\text {odd }}^{\text {alt }}}{E_{\text {even }}^{\text {alt }}}$.

Theorem 3.10. (Carlitz) Let $\delta_{n}=(n-1, n-2, \ldots, 1), n \geq 2$, be the staircase shape. (Set $\delta_{1}=\emptyset$.) Then

$$
\begin{aligned}
\frac{E_{\text {odd }}^{\text {alt }}}{E_{\text {even }}^{\text {alt }}} & =s_{(1)}+\sum_{n \geq 3} s_{\delta_{n} / \delta_{n-2}} \\
& =\tan \left(\sum_{i \geq 1} \arctan x_{i}\right) .
\end{aligned}
$$

Carlitz' proof that $\frac{E_{\text {odd }}^{\text {alt }}}{E_{\text {even }}^{\text {alt }}}$ equals (3.11) is recovered in [1, Proof of Proposition 3.4], in the language of symmetric functions and skew tableaux.

Let $E_{n}$ be the $n$th Euler number; $E_{n}$ is the cardinality of the set of alternating (downup) permutations $\left\{\sigma \in S_{n}: \sigma(1)>\sigma(2)<\sigma(3)>\ldots\right\}$. Note that $E_{2 n+1}$ is the tangent number which appeared in Proposition 2.6 and Proposition 3.7. Also let $z_{\lambda}$ denote the order of the centraliser of an element of $S_{n}$ with cycle-type $\lambda \vdash n$; thus $z_{\lambda}=\prod_{i} i^{m_{i}} m_{i}$ ! if $\lambda$ has $m_{i}$ parts equal to $i$. The following theorem is due to Foulkes [4]. See also [10].

Theorem 3.11. (Foulkes) Let $\delta_{n}=(n-1, n-2, \ldots, 1)$. Then

$$
s_{\delta_{n} / \delta_{n-2}}=\sum_{\substack{\lambda \vdash 2 n-3 \\ \lambda \text { has only odd parts }}}(-1)^{\frac{n-\ell(\lambda)}{2}} E_{\ell(\lambda)} z_{\lambda}^{-1} p_{\lambda}
$$

In particular the dimension of the representation indexed by the skew shape $\delta_{n} / \delta_{n-2}$ is the Euler number $E_{n}$.

We now deduce a similar skew-Schur function expansion for the quotient appearing in the left side of Theorem 1.1.

Theorem 3.12. Let $\delta_{n}=(n-1, n-2, \ldots, 1), n \geq 2$. (Set $\delta_{1}=\emptyset$.) Then

$$
\begin{aligned}
\frac{E_{\text {odd }}}{E_{\text {even }}} & =s_{(1)}+\sum_{n \geq 3}(-1)^{n} s_{\delta_{n} / \delta_{n-2}} \\
& =\tanh \left(\sum_{i \geq 1} \operatorname{arctanh} x_{i}\right) .
\end{aligned}
$$

If $H k_{n}$ is the sum of all Schur functions indexed by hooks of size $n$, then

$$
(-1)^{n-2} s_{\delta_{n} / \delta_{n-2}}=\sum_{\substack{\mu \vdash(2 n-1) \\
\mu=\prod i^{m_{i}}}}(-1)^{\ell(\mu)-1}\left(\begin{array}{c}
\ell(\mu) \\
m_{1}, m_{2}, \ldots
\end{array}\right) H k_{1}^{m_{1}} H k_{2}^{m_{2}} \ldots
$$


Proof. Rewrite Carlitz' result, Theorem 3.10, as a generating function, noting that $s_{\delta_{n} / \delta_{n-2}}$ has degree $1+2(n-2)$.

$$
\frac{E_{\text {odd }}^{\text {alt }}(t)}{E_{\text {even }}^{\text {alt }}(t)}=t s_{(1)}+\sum_{n \geq 3} t t^{2(n-2)} s_{\delta_{n} / \delta_{n-2}}
$$

Clearly the substitution $t \rightarrow i t$ converts the left-hand side to $\frac{i E_{\text {odd }}(t)}{E_{\text {even }}(t)}$ and the right-hand side to

$$
i\left(t s_{(1)}+\sum_{n \geq 3} t^{2 n-3}(-1)^{n-2} s_{\delta_{n} / \delta_{n-2}}\right) .
$$

Similarly, rewrite the second equality in Theorem 3.10 as a generating function, giving:

$$
\frac{E_{\text {odd }}^{\text {alt }}(t)}{E_{\text {even }}^{\text {alt }}(t)}=\tan \left(\sum_{j \geq 1} \arctan \left(t x_{j}\right)\right)
$$

and make the same substitution $t \rightarrow i t$, noting that

$$
\arctan (i z)=i \operatorname{arctanh}(z), \tan (i x)=i \tanh (x) \text {. }
$$

Alternatively, it is easy to show directly that $\mathcal{E}=\frac{E_{\text {odd }}}{E_{\text {even }}}$ satisfies the same recurrence as the hyperbolic tangent function, namely

$$
\tanh (x+y)=\frac{\tanh (x)+\tanh (y)}{1+\tanh (x) \tanh (y)}
$$

Indeed, writing $f(x \geq m)$ for the function $f\left(x_{m}, x_{m+1}, \ldots\right)$, it can be verified that

$$
\mathcal{E}(x \geq 1)=\frac{x_{1}+\mathcal{E}(x \geq 2)}{1+x_{1} \mathcal{E}(x \geq 2)}
$$

Finally, the expression (3.14) follows by using Part (2) of Proposition 2.6.

We observe two equivalent identities that are consequences of Propositions 2.6 and 3.7. and equations (3.11) and (3.13). By (3.17) the two identities (3.18) and (3.20) are equivalent, and also follow from the power series expansion of the arctangent function.

\section{Corollary 3.13.}

$$
\sum_{j \geq 1} \arctan t x_{j}=\sum_{k \geq 0} \frac{(-1)^{k} p_{2 k+1} t^{2 k+1}}{2 k+1}
$$

and hence

$$
\begin{gathered}
\left(\sum_{j \geq 1} \arctan x_{j}\right)[\text { Lie odd }]=\arctan p_{1} . \\
\sum_{j \geq 1} \operatorname{arctanh} t x_{j}=\sum_{k \geq 0} \frac{p_{2 k+1} t^{2 k+1}}{2 k+1}
\end{gathered}
$$

and hence

$$
\left(\sum_{j \geq 1} \operatorname{arctanh} x_{j}\right)\left[\text { Lie }_{\text {odd }}\right]=\operatorname{arctanh} p_{1} .
$$


Remark 3.14. From Eqn. (2.3), we see that the odd Lie characteristics Lie $_{\text {odd }}$ belong to the $\mathbb{Q}$-subalgebra of symmetric functions generated by the odd power sums $\left\{p_{2 k+1}, k \geq 0\right\}$. The expansions for $\tanh (x)$ and $\tan (x)$ in Part (3) of Proposition 3.7 and Proposition 2.6 respectively, show that the same is true for the quotients $\frac{H_{\text {odd }}}{H_{\text {even }}}=\frac{E_{\text {odd }}}{E_{\text {even }}}$ and $\frac{H_{\text {odd }}^{\text {alt }}}{H_{\text {even }}^{\text {alt }}}=\frac{E_{\text {odd }}^{\text {alt }}}{E_{\text {even }}^{\text {alt }}}$, and similarly for the product $H E$, by expanding the exponential in Part (4) of Proposition 2.2 as a product of power series in $p_{2 k+1} /(2 k+1)$. See also [5. Chapter III, Section 8, Eqn. (8.5)] and [9, Exercise 19]. A theorem of Ardila and Serrano [1, Theorem 4.3] asserts that the staircase Schur function $s_{\delta_{n} / \delta_{n-2}}$, and hence the quotients $E_{\text {odd }}^{\text {alt }} / E_{\text {even }}^{\text {alt }}$, have a positive expansion in terms of Schur $P$-functions, which form a basis for $\mathbb{Q}\left[p_{2 k+1}, k \geq 0\right]$.

We close by mentioning one other context known to us, in which the odd Lie representations arise. Consider the $S_{n}$-representation $\eta_{n}$ on the multilinear component of the free Jordan algebra with $n$ generators. The Jordan algebra has a bracket defined by $[x, y]=x y+y x$. The $S_{n}$-module afforded by this bracket and its deformations were determined in [3]. The main theorem of [3] implies that the $S_{n}$-representation on the free Jordan algebra is determined by symmetrising $L_{i} e_{o d d}$. Recall that $H=\sum_{n \geq 0} h_{n}$.

Theorem 3.15. [3, Theorem 2.1] Define $\eta_{0}=1$. The following plethystic identity holds:

$$
\sum_{n \geq 0} \eta_{n}=H\left[\text { Lie }_{\text {odd }}\right]
$$

Clearly this equation can be decomposed according to parity to give plethystic expressions for $\sum_{n \geq 0, n \equiv i(\bmod 2)} \eta_{n}, i=0,1$.

Acknowledgments. I am grateful to Richard Stanley for communicating his conjecture, and for supplying pertinent references. I also wish to convey my gratitude to the anonymous referees for a careful reading of the paper, and for comments leading to valuable improvements.

\section{REFERENCES}

1. F. Ardila and L.G. Serrano, Staircase skew Schur functions are Schur P-positive, J. Algebraic Combin. 36 (2012), 409-423. DOI 10.1007/s10801-012-0342-8

2. L. Carlitz, Enumeration of up-down sequences, Discrete Math. 4 (1973), 273-286.

3. R. Calderbank, P. Hanlon and S. Sundaram, Representations of the symmetric group in deformations of the free Lie algebra, Trans. Amer. Math. Soc. 341, 1 (1994), 315-333.

4. H. O. Foulkes, Enumeration of permutations with prescribed up-down and inversion sequences, Discrete Math. 15 (1976), 235-252.

5. I. G. Macdonald, Symmetric functions and Hall polynomials, Second Edition, Oxford University Press (1995).

6. C. Reutenauer, Free Lie Algebras, London Mathematical Society Monographs, Oxford Science Publications (1993).

7. R. P. Stanley, Enumerative Combinatorics, Vol.1, With a foreword by Gian-Carlo Rota. Corrected reprint of the 1986 original. Cambridge Studies in Advanced Mathematics, 49. Cambridge University Press, Cambridge, 1997.

8. R. P. Stanley, Enumerative Combinatorics, Vol.2, Cambridge Studies in Advanced Mathematics 62, Cambridge University Press, Cambridge, 1999. 
9. R. P. Stanley, Supplementary Exercises for Chapter 7 of Enumerative Combinatorics, Vol. 2, 2014.

10. R. P. Stanley, Alternating permutations and symmetric functions, J. Combin. Theory Ser. A 114 (2007), no. 3, 436-460.

11. S. Sundaram, Applications of the Hopf trace formula to computing homology representations, Jerusalem Combinatorics '93, 277-309, Contemp. Math., 178, Amer. Math. Soc., Providence, RI, 1994.

12. S. Sundaram, The conjugacy action of $S_{n}$ and modules induced from centralisers, J. Algebraic Combin. 48 (2018), no. 2, 179-225. DOI 10.1007/s10801-017-0796-9

13. S. Sundaram, Variations on the $S_{n}$-module Lie $_{n}$, arXiv.math.RT/1803.09368.

14. S. Sundaram, On a curious variant of the $S_{n}$-module Lie ${ }_{n}$, Algebr. Comb. 3 (2020), no. 4, 9851009. https://doi.org/10.5802/alco.127

15. R. M. Thrall, On symmetrized Kronecker powers and the structure of the free Lie ring, Amer. J. Math. 64 (1942), 371-388.

Pierrepont School, One Sylvan Road North, Westport, CT 06880

Email address: shsund@comcast.net 